\title{
On the Kähler manifolds with the largest infimum of spectrum of Laplace-Beltrami operators and sharp lower bound of Ricci or holomorphic bisectional curvatures
}

\author{
SONG-YING LI
}

\begin{abstract}
The paper studies the extremal or rigidity problem associated to the largest infimum of spectrum of Laplace-Beltrami operator $\Delta_{g}$ on Kähler manifolds $\left(M^{n}, g\right)$ under the sharp lower bound assumption on either Ricci curvature or holomorphic bisectional curvature. The paper provides some conterexamples on those rigidity problems. In particular, we consider $D(A)=\left\{z \in \mathbb{C}^{n}:|z|^{2}+\right.$ $\left.\operatorname{Re} \sum_{j=1}^{n} A_{j} z_{j}^{2}<1\right\}$ a convex domain in $\mathbb{C}^{n}$ with $n>1$ and $A_{j} \in$ $(-1,1)$. Assuming $g_{0}$ is the Kähler-Einstein metric on $D(A)$, we prove that $\lambda_{1}\left(\Delta_{g_{0}}\right)=n^{2}$ on $\left(D(A), g_{0}\right)$, but $D(A)$ is not biholomorphic to the unit ball $B_{n}$ when $A \neq 0$. Moreover, we prove that $\rho(z)=-e^{u}$ is strictly plurisubharmonic in $D(A)$ where $u$ is the potential function for Kähler-Einstein metric on $D(A)$. We also construct a complete Kähler metric $g_{1}$ on $D(A)$ with holomorphic bisectional curvature $\mathcal{K}_{g_{1}} \geq-1$ and $\lambda_{1}\left(\Delta_{g_{1}}\right)=n^{2}$, but $D(A)$ is not biholomorphic to $B_{n}$ when $A \neq 0$.
\end{abstract}

\section{Introduction}

Let $\left(M^{n}, g\right)$ be a Kähler manifold of complex dimension $n$ with Kähler metric $g=\sum_{i, j=1}^{n} g_{i \bar{j}} d z_{i} \otimes d \bar{z}_{j}$. Then the Laplace-Beltrami operator with respect to the metric $g$ is defined as follows:

$$
\Delta_{g}=-4 \sum_{i, j=1}^{n} g^{i \bar{j}} \frac{\partial^{2}}{\partial z_{i} \partial \bar{z}_{j}}
$$

where $\left[g^{i \bar{j}}\right]^{t}=\left[g_{i \bar{j}}\right]^{-1}$. We define

$$
\lambda_{1}\left(\Delta_{g}, M\right)=\inf \left\{4 \int_{M} \sum_{i, j=1}^{n} g^{i \bar{j}} \frac{\partial f}{\partial z_{i}} \frac{\partial f}{\partial \bar{z}_{j}} d v_{g}: f \in C_{0}^{\infty}(M),\|f\|_{L^{2}}=1\right\},
$$


where $d v_{g}$ is the volume measure on $M$ with respect to the Kähler metric $g$.

When $M$ is compact and $\Delta_{g}$ is uniformly elliptic, $\lambda_{1}\left(\Delta_{g}\right)$ is the first positive eigenvalue of $\Delta_{g}$ with Dirichlet boundary condition (see, for example, the lecture notes of $\mathrm{Li}[8]$ and the paper of S. Udagawa [19] and references therein). When $\left(M^{n}, g\right)$ is a complete noncompact Kähler manifold, in general, $\lambda_{1}\left(\Delta_{g}\right)$ is not an eigenvalue of $\Delta_{g}$ (see for example, [1, 10, 11, $16,18])$. However, it is the infimum of the positive spectrum of $\Delta_{g}$. There have been many researches on the analytic and geometric problems associated with $\lambda_{1}$. An important analytic problem is to find an sharp estimate on $\lambda_{1}\left(\Delta_{g}\right)$ with certain low bound assumptions on curvatures, which provides sharp upper bound for Poincarè inequality. An important upper bound estimate was obtained by $\mathrm{Li}$ and Wang in [10]. They proved that $\lambda_{1}\left(\Delta_{g}\right) \leq n^{2}$ if the holomorphic bisectional curvature of $M$ is bounded below by -1 . Their estimate is sharp and equality is achieved by the complex hyperbolic space $\mathrm{CH}^{n}$. Cheng [1] proved that $\lambda_{1}\left(\Delta_{g}\right) \leq n^{2}$ if Ricci curvature satisfying $R_{i \bar{j}} \geq-n g_{i \bar{j}}$. Munteanu [18] proved that $\lambda_{1}\left(\Delta_{g}\right) \leq n^{2}$ if the Ricci curvature $R_{i \bar{j}} \geq-(n+1) g_{i \bar{j}}$. His estimate is sharp because if $M=B_{n}$ and $g$ is Kähler-Einstein metric then $\lambda_{1}=n^{2}$ and $R_{i \bar{j}}=-(n+1) g_{i \bar{j}}$. Some more results along this line with different assumptions and estimates were obtained in $[5,6,11,21]$ and references therein. Li and Tran [16] provided some alternative conditions; the authors considered a bounded pseudoconvex domain $D$ in $\mathbb{C}^{n}$ with negative defining function $\rho$ so that $u=-\log (-\rho)$ is strictly plurisubharmonic in $D$, and Kähler metric $g_{i \bar{j}}=\frac{\partial^{2} u}{\partial z_{i} \partial \bar{z}_{j}}$ (induced by $u$ ). They proved several theorems. In particular, the following result was proved in [16] which will be used later.

Theorem 1.1. Let $D$ be a bounded strictly pseudoconvex domain in $\mathbb{C}^{n}$ with a plurisubharmonic negative defining function $\rho \in C^{2}(\bar{D})$ so that $u=$ $-\log (-\rho)$ is strictly plurisubharmonic in D. Let $g$ be the Kähler metric induced by $u$. Then $(D, g)$ is a complete Kähler manifold and $\lambda_{1}\left(\Delta_{g}\right)=n^{2}$.

Geometric-type problems associated with $\lambda_{1}\left(\Delta_{g}\right)$ are rigidity problems. One may raise the following general questions:

Question 1.1. Under the assumptions: holomorphic bisectional curvature $\mathcal{K}_{g} \geq-1$ and $\lambda_{1}\left(\Delta_{g}\right)=n^{2}$. What can one say about $M$ ?

Question 1.2. Under the assumptions: Ricci curvature $R_{i \bar{j}} \geq-(n+1) g_{i \bar{j}}$ and $\lambda_{1}\left(\Delta_{g}\right)=n^{2}$. What can one say about $M$ ? 
Questions 1.1 and 1.2 for the Riemannian case were studied by Li and Wang $[11,13]$, and they proved a very pretty splitting theorem. Li and Wang [10, 12] considered Kähler manifolds and also obtained a similar splitting theorem. The following theorem is their results for the Kähler case.

Theorem 1.2. Let $\left(M^{n}, g\right)$ be a complete Kähler manifold. Then

(i) If the Ricci curvature $R_{i \bar{j}} \geq-(n+1) g_{i \bar{j}}$ and $\lambda_{1}>\frac{n+1}{2}$, then $M$ must have one infinite volume end;

(ii) If the holomorphic bisectional curvature $\mathcal{K}_{g} \geq-1$ and $\lambda_{1}=n^{2}$, then either $M$ has only one end or $M=\mathbb{R} \times N$ with $N$ being a compact manifold. Moreover, the metric on $M$ is of the form

$$
d s_{M}^{2}=d t^{2}+\mathrm{e}^{4 t} \omega_{2}^{2}+\mathrm{e}^{2 t} \sum_{i=3}^{2 n} \omega_{i}^{2},
$$

where $\left\{\omega_{2}, \ldots, \omega_{2 n}\right\}$ are orthonormal basis of $N$ with $J d t=\omega_{2}$.

Munteanu [18] proved the same result under a weaker condition: $R_{i \bar{j}} \geq$ $-(n+1) g_{i j}$ and $\lambda_{1}=n^{2}$. Kong et al. [6] considered a complete quaternonic Kähler manifold $\left(M^{4 n}, g\right)$ and proved the same theorem under the condition: the scalar curvature $S_{M} \geq-16 n(n+2)$ and $\lambda_{1} \geq(2 n+1)^{2}$.

As we know that if $M=B_{n}$ is the unit ball in $\mathbb{C}^{n}$ and $g$ is the KählerEinstein metric then

$$
\lambda_{1}\left(\Delta_{g}\right)=n^{2}, \quad R_{i \bar{j}}=-(n+1) g_{i \bar{j}}, \quad R_{i \bar{j} k \bar{\ell}}=g_{i \bar{j}} g_{k \bar{\ell}}+g_{k \bar{j}} g_{i \bar{\ell}}
$$

which means that holomorphic bisectional curvature equals -1 . Comparing Obata theorem and Cheng theorem for compact Riemannian manifolds (see $[8,9])$ and result in $[15]$. It is natural to ask the following question.

Question 1.3. Assume that $D$ is a smoothly bounded strictly pseudoconvex domain in $\mathbb{C}^{n}$ with a complete Kähler metric $g$ satisfying either

$$
R_{i \bar{j}}=-(n+1) g_{i \bar{j}}
$$

or holomorphic bisectional curvature $\mathcal{K}_{g} \geq-1$. Assume that $\lambda_{1}\left(\Delta_{g}\right)=n^{2}$. Is $D$ biholomorphic to the ball in $\mathbb{C}^{n}$ ?

The main purpose of this paper is to provide a way of constructing some counter examples for Question 1.3. In particular, we consider strictly a 
pseudoconvex domain in $\mathbb{C}^{n}$ whose boundary is real ellipsoid. After a linearly holomorphic change of variables, these domains can be described as follows:

$$
D(A)=\left\{z \in \mathbb{C}^{n}: r(z)=|z|^{2}+\operatorname{Re} \sum_{j=1}^{n} A_{j} z_{j}^{2}-1<0\right\}
$$

with

$$
0 \leq A_{1} \leq A_{2} \leq \cdots \leq A_{n}<1
$$

It was proved by Webster [22] that $D(A)$ is biholomorphic to the unit ball in $\mathbb{C}^{n}$ if and only if $A=\left(A_{1}, \ldots, A_{n}\right)=0$. We will prove the following theorem in this paper.

Theorem 1.3. For any $0 \leq A_{1} \leq \cdots \leq A_{n}<1$, we have the following:

(i) If $g$ is the Kähler-Einstein metric on $D(A)$ then $\lambda_{1}\left(\Delta_{g}\right)=n^{2}$.

(ii) There is a Kähler metric $g^{0}$ on $D(A)$ with $A_{n} \leq 2 / 5$ so that the holomorphic bisectional curvature $\mathcal{K}_{g^{0}} \geq-1$ and $\lambda_{1}\left(\Delta_{g_{0}}\right)=n^{2}$.

Combining Theorem 1.3 and the above theorem of Webster in [22], we answer Question 1.3 negatively with the counter examples: $D(A)$ with $A \neq 0$ and $n>1$.

This paper is organized as follows: in Section 2, we provide an explicit formula to approximate the potential function of the Kähler-Einstein metric. In Section 3, we prove part (i) of Theorem 1.3. Finally, part (ii) of Theorem 1.3 is proved in Section 4.

\section{Approximation formula}

Let $D$ be a smoothly bounded pseudoconvex domain in $\mathbb{C}^{n}$. A plurisubharmonic function $u$ on $D$ is said to be the potential function for the KählerEinstein metric on $D$ if $u$ is the solution of the Monge-Ampère equation:

$$
\operatorname{det} H(u)=e^{(n+1) u} \quad \text { in } D ; \quad u=+\infty \quad \text { on } \partial D
$$

The existence of such a solution for (2.1) was proved by Cheng and Yau [2], the uniqueness was proved by Fefferman [3]. When $D$ is strictly pseudoconvex, Cheng and Yau [2] prove that $\mathrm{e}^{-u} \in C^{n+3 / 2}(\bar{D})$. Lee and Melrose [7] 
give the following asymptotic expansion for $\mathrm{e}^{-u}$ :

$$
\rho(z)=-e^{-u(z)}=\sum_{j=0}^{\infty} a_{j} \rho^{0}(z)\left(\rho^{0}(z)^{n+1} \log \left(-\rho^{0}(z)\right)^{j}\right.
$$

where $a_{j} \in C^{\infty}(\bar{D})$ and $\rho^{0}$ is any negative defining function for $D$ with $\rho^{0} \in$ $C^{\infty}(\bar{D})$. Fefferman [3] gave a method of how to approximate $\rho(z)$ in terms of $\rho^{0}(z)$. In particular, he proved $a_{0}(z)=J\left(\rho^{0}\right)^{-1 /(n+1)}+\sum_{j=1}^{n} a_{0, j}\left(\rho^{0}(z)\right)^{j}$, where

$$
J\left(\rho_{0}\right)(z)=-\operatorname{det}\left[\begin{array}{cc}
\rho_{0} & \bar{\partial} \rho_{0}(z) \\
\left(\bar{\partial} \rho_{0}\right)^{*} & H\left(\rho_{0}\right)
\end{array}\right]
$$

where $\bar{\partial} \rho_{0}=\left(\frac{\partial \rho_{0}}{\partial \bar{z}_{1}}, \ldots, \frac{\partial \rho_{0}}{\partial \bar{z}_{n}}\right), H\left(\rho^{0}\right)$ is the complex Hessian matrix of $\rho_{0}$ on $D$. Question about how to compute $a_{0, j}$ in (2.2) in terms of $\rho^{0}$ explicitly has been studied by Fefferman [3] and Graham [4]; they provided a certain iteration formula for evaluating $a_{0, j}$, respectively. Here we give an alternative formula for $a_{0, j}$ or approximation $\rho$ in terms of $\rho^{0}$ as follows.

Theorem 2.1. Let $r(z)$ be a smooth negative defining function for $D$ so that $\ell(\rho):=-\log (-r(z))$ is strictly plurisubharmonic in D. Let

$$
\rho_{0}(z)=r(z), \quad \rho_{j+1}(z)=\rho_{j}(z) J\left(\rho_{j}\right)^{-1 /(n+1)} \mathrm{e}^{-B_{j}}
$$

with

$$
B_{j}(z)=\frac{\operatorname{tr}\left(H\left(\ell\left(\rho_{j}\right)\right)^{-1} H\left(\log J\left(\rho_{j}\right)\right)\right.}{(j+2)(n-j)(n+1)} .
$$

Then

$$
J\left(\rho_{j+1}\right)(z)=1+O\left(\delta(z)^{j+2}\right), \quad j=0,1, \ldots, n-1
$$

and

$$
\delta(z)=\operatorname{dist}(z, \partial D), \quad a_{0}(z)=\frac{\rho_{n}(z)}{\rho_{0}(z)}
$$

Moreover, if

$$
B_{n}=\frac{\operatorname{tr}\left(H\left(\ell\left(\rho_{n}\right)\right)^{-1} H\left(\log J\left(\rho_{n}\right)\right)\right)}{(n+2)(n+1)} \ell\left(\rho_{n}(z)\right)
$$


then

$$
J\left(\rho_{n+1}\right)=1+O\left(\delta(z)^{n+2} \log \delta(z)\right) .
$$

Proof. Let $K=J\left(\rho_{j}\right) \exp \left((n+1) b_{j} \rho_{j}\right)$ with $B_{j}=b_{j} \rho_{j}(z)$. Then $\rho_{j+1}=\rho_{j}$ $K^{-1 /(n+1)}$ and

$$
\begin{aligned}
J\left(\rho_{j+1}\right) & \\
= & \left(-\rho_{j+1}(z)\right)^{n+1} \operatorname{det} H\left(\ell\left(\rho_{j+1}\right)\right)(z) \\
= & \frac{J\left(\rho_{j}\right)}{K} \operatorname{det}\left[I_{n}+\frac{1}{(n+1)} H\left(\ell\left(\rho_{j}\right)\right)^{-1} H(\log K)(z)\right] \\
= & \mathrm{e}^{-(n+1) B_{j}} \operatorname{det}\left[I_{n}+\frac{1}{n+1} H\left(\ell\left(\rho_{j}\right)\right)^{-1}\left(H\left(\log J\left(\rho_{j}\right)\right)(z)+(n+1) H\left(b_{j} \rho_{j}\right)\right)\right] \\
= & \mathrm{e}^{-(n+1) B_{j}} \operatorname{det}\left[I_{n}+\frac{1}{n+1} H\left(\ell\left(\rho_{j}\right)\right)^{-1}\left(H\left(\log J\left(\rho_{j}\right)\right)(z)\right.\right. \\
& \left.+H\left(\ell\left(\rho_{j}\right)\right)^{-1}\left(b_{j} H\left(\rho_{j}\right)+\left(\bar{\partial} b_{j}\right)^{*}\left(\bar{\partial} \rho_{j}\right)+\left(\bar{\partial} \rho_{j}\right)^{*}\left(\bar{\partial} b_{j}\right)+\rho_{j}(z) H\left(b_{j}\right)\right)\right] .
\end{aligned}
$$

Let $X=\left[x_{i j}\right]$ be an $n \times n$ matrix . Then

$$
\operatorname{det}\left[I_{n}+X\right]=1+\operatorname{tr}(X)+O(\delta(z)), \quad \text { if } \quad x_{i j}=O(\delta(z)) .
$$

Notice that

$$
\begin{aligned}
& \sum_{j=1}^{n}\left(\rho^{i \bar{j}}-\frac{\rho^{i} \rho^{\bar{j}}}{|\partial \rho|_{\rho}^{2}-\rho}\right) \partial_{\bar{j}} \rho=\frac{\rho^{i}(-\rho)}{|\partial \rho|_{\rho}^{2}-\rho} \\
& \quad \times \sum_{i, j=1}^{n}\left(\rho^{i \bar{j}}-\frac{\rho^{i} \rho^{\bar{j}}}{|\partial \rho|_{\rho}^{2}-\rho}\right) \partial_{\bar{j}} \rho \partial_{i} \rho=\frac{|\partial \rho|_{\rho}^{2}(-\rho)}{|\partial \rho|_{\rho}^{2}-\rho}
\end{aligned}
$$

one has

$$
\begin{aligned}
& \operatorname{tr}\left(H\left(\ell\left(\rho_{0}\right)\right)^{-1}\left(b_{0} H\left(\rho_{0}\right)+\left(\bar{\partial} b_{0}\right)^{*}\left(\bar{\partial} \rho_{0}\right)+\left(\bar{\partial} \rho_{0}\right)^{*}\left(\bar{\partial} b_{0}\right)+\rho_{0}(z) H\left(b_{0}\right)\right)\right) \\
& \quad=-(n-1) b_{0} \rho_{0}(z)+O\left(\delta(z)^{2}\right) .
\end{aligned}
$$

If we let

$$
b_{0}(z) \rho_{0}(z)=B_{0}(z)=\frac{1}{2 n(n+1)} \operatorname{tr}\left(H\left(\ell\left(\rho_{0}\right)\right)^{-1} H\left(\log J\left(\rho_{0}\right)\right)(z),\right.
$$


then by (2.10), (2.12) and (2.13), one has

$$
\begin{aligned}
J\left(\rho_{1}\right)(z)=1 & +\frac{\operatorname{tr}\left(H\left(\ell\left(\rho_{0}\right)\right)^{-1} H\left(\log J\left(\rho_{0}\right)\right)(z)\right.}{(n+1)} \\
- & (n-1) b_{0} \rho_{0}(z)-(n+1) B_{0}(z)+O\left(\delta(z)^{2}\right) \\
& =1+O\left(\delta(z)^{2}\right) .
\end{aligned}
$$

Assume that $J\left(\rho_{j}\right)=1+O\left(\delta(z)^{j+1}\right)$ for some $1 \leq j \leq n-1$. Then by $(2.12)$, one has

$$
\operatorname{tr}\left(H\left(\ell\left(\rho_{j}\right)\right)^{-1} H\left(\log J\left(\rho_{j}\right)\right)=O\left(\delta(z)^{j+1}\right) .\right.
$$

If one assumes (a priori) that

$$
B_{j}(z)=b_{j}(z) \rho_{j}(z)=\tilde{b_{0}}\left(\rho_{j}(z)\right)^{j+1}, \quad \tilde{b_{0}} \in C^{\infty}(\bar{D})
$$

and uses (2.12), then one has

$$
\begin{aligned}
& \operatorname{tr}\left(H\left(\ell\left(\rho_{j}\right)\right)^{-1}\left(b_{j} H\left(\rho_{j}\right)+\left(\bar{\partial} b_{j}\right)^{*}\left(\bar{\partial} \rho_{j}\right)+\left(\bar{\partial} \rho_{j}\right)^{*}\left(\bar{\partial} b_{j}\right)+\rho_{j}(z) H\left(b_{j}\right)\right)\right) \\
& \quad=-(n-1) B_{j}(z)+2 j B_{j}-j(n-j) B_{j}+O\left(\delta(z)^{n+2}\right) .
\end{aligned}
$$

Similar to (2.15), if we let

$$
B_{j}=\frac{\operatorname{tr}\left(H\left(\ell\left(\rho_{j}\right)\right)^{-1} \log J\left(\rho_{j}\right)\right)}{(2+j)(n-j)(n+1)}
$$

then

$$
J\left(\rho_{j+1}\right)=1+O\left(\delta(z)^{j+2}\right)
$$

for $j=1,2, \ldots, n-1$.

Remark. We must notice here that in the proof of (2.12), we use $H(\rho)$ as positive definite near $\partial D$. When $H\left(\rho_{j}\right)$ is not strictly positive definite, the conclusion remains true with the following argument: let $\tilde{\rho}_{j}=\rho_{j}+C \rho_{j}^{2}$ with 
some positive number $C$ so that $H\left(\tilde{\rho}_{j}\right)$ is positive definite near $\partial D$. Then

$$
\begin{aligned}
H\left(\ell\left(\rho_{j}\right)\right)^{-1} & =(1+c \rho)^{-1}\left[H\left(\ell\left(\tilde{\rho}_{j}\right)\right)+\frac{c(1+2 c \rho)}{1+c \rho}\left(\partial \rho_{j}\right) \otimes\left(\bar{\partial} \rho_{j}\right)\right]^{-1} \\
& =\left(1+O(\delta(z)) H\left(\ell\left(\tilde{\rho}_{j}\right)^{-1}\right.\right.
\end{aligned}
$$

With the help of the above formula, all arguments in proof of (2.12) remain true.

When $j=n$, if we let

$$
\rho_{n+1}(z)=\rho_{n} J\left(\rho_{n}\right)^{-1 /(n+1)} \mathrm{e}^{-B_{n}(z)}, \quad B_{n}(z)=b_{n} \rho_{n}(z)\left(-\log \left(-\rho_{n}(z)\right),\right.
$$

then

$$
\begin{aligned}
& J\left(\rho_{n+1}\right)=\mathrm{e}^{-(n+1) B_{n}} \operatorname{det}\left[I_{n}+\frac{1}{n+1} H\left(\ell\left(\rho_{n}\right)\right)^{-1}\left(H\left(\log J\left(\rho_{n}\right)\right)(z)\right.\right. \\
& \left.+(n+1) H\left(b_{n} \rho_{n} \ell\left(\rho_{n}\right)\right)\right] \\
& =\mathrm{e}^{-(n+1) B_{n}} \operatorname{det}\left[I_{n}+\frac{1}{n+1} H\left(\ell\left(\rho_{n}\right)\right)^{-1} H\left(\log J\left(\rho_{n}\right)\right)(z)\right. \\
& +\ell\left(\rho_{n}\right) H\left(\ell\left(\rho_{n}\right)\right)^{-1}\left(b_{n} H\left(\rho_{n}\right)+\left(\bar{\partial} b_{n}\right)^{*}\left(\bar{\partial} \rho_{n}\right)+\left(\bar{\partial} \rho_{n}\right)^{*}\left(\bar{\partial} b_{n}\right)\right. \\
& \left.+\rho_{n}(z) H\left(b_{n}\right)\right)+H\left(\ell\left(\rho_{n}\right)\right)^{-1}\left(\rho _ { n } ( z ) b _ { n } H \left(\ell\left(\rho_{n}\right)-\left(\bar{\partial} b_{n}\right)^{*}\left(\bar{\partial} \rho_{n}\right)\right.\right. \\
& \left.\left.\left.-\left(\bar{\partial} \rho_{n}\right)^{*}\left(\bar{\partial} b_{n}\right)\right)+\frac{2 b_{n}}{-\rho_{n}}\left(\bar{\partial} \rho_{n}\right)^{*}\left(\bar{\partial} \rho_{n}\right)\right]\right] \\
& =\mathrm{e}^{-(n+1) B_{n}} \operatorname{det}\left[I_{n}+\frac{1}{n+1} H\left(\ell\left(\rho_{n}\right)\right)^{-1} H\left(\log J\left(\rho_{n}\right)\right)(z)\right. \\
& +\ell\left(\rho_{n}\right) b_{n}(n+1) H\left(\ell\left(\rho_{n}\right)\right)^{-1}\left(H\left(\rho_{n}\right)+\frac{n}{\rho_{n}(z)}\left(\bar{\partial} \rho_{n}\right)^{*}\left(\bar{\partial} \rho_{n}\right)\right] \\
& +\ell\left(\rho_{n}\right) H\left(\ell\left(\rho_{n}\right)\right)^{-1}\left(( \rho _ { n } ( z ) ) ^ { n } ( n + 1 ) \left[\left(\bar{\partial} \tilde{b}_{n}\right)^{*}\left(\bar{\partial} \rho_{n}\right)\right.\right. \\
& \left.\left.\left.+\left(\bar{\partial} \rho_{n}\right)^{*}\left(\bar{\partial} \tilde{b}_{n}\right)\right]+\left(\rho_{n}(z)\right)^{n+1} H\left(\tilde{b}_{n}\right)\right)\right] \\
& +H\left(\ell\left(\rho_{n}\right)\right)^{-1}\left[\rho_{n}(z) b_{n} H\left(\ell\left(\rho_{n}\right)+2(n+1) \frac{b_{n}}{-\rho_{n}}\left(\bar{\partial} \rho_{n}\right)^{*}\left(\bar{\partial} \rho_{n}\right)\right]\right. \\
& \left.\left.-H\left(\ell\left(\rho_{n}\right)\right)^{-1}\left[\rho_{n}(z)\right)^{n}\left(\left(\bar{\partial} \tilde{b}_{n}\right)^{*}\left(\bar{\partial} \rho_{n}\right)+\left(\bar{\partial} \rho_{n}\right)^{*}\left(\bar{\partial} \tilde{b}_{n}\right)\right)\right]\right] \\
& =1+O\left(\delta(z)^{n+2} \log (\delta(z))\right)
\end{aligned}
$$


if we choose

$$
b_{n}(z) \rho_{n}(z)=\frac{1}{(n+2)(n+1)} \operatorname{tr}\left(H\left(\ell\left(\rho_{n}\right)\right)^{-1} H\left(\log J\left(\rho_{n}\right)\right)\right) .
$$

This completes the proof of Theorem 2.1.

\section{Domain whose boundary is real ellipsoid}

We consider a class of strictly pseudoconvex domains in $\mathbb{C}^{n}$ with the real ellipsoid as their boundary. After a linearly holomorphic change of variables, those domains can be written as

$$
D(A)=\left\{z \in \mathbb{C}^{n}: r(z)<0\right\}
$$

where

$$
r(z)=|z|^{2}+\operatorname{Re} \sum_{j=1}^{n} A_{j} z_{j}^{2}-1, \quad 0 \leq A_{1} \leq A_{2} \leq \cdots \leq A_{n}<1 .
$$

Then $H(r)=I_{n}$ and

$$
J(r)=-r(z)+\sum_{j=1}^{n}\left|r_{j}(z)\right|^{2}=\operatorname{Re} \sum_{j=1}^{n} A_{j} z_{j}^{2}+\sum_{j=1}^{n} A_{j}^{2}\left|z_{j}\right|^{2}+1 .
$$

Let

$$
\rho^{1}(z)=\mathrm{e}^{-b_{0}(z) r(z)} J(r)^{-\frac{1}{n+1}} r(z),
$$

where

$$
b_{0}(z) r(z)=\frac{1}{2 n(n+1)} \operatorname{tr}\left(H(\ell(r))^{-1} H(\log J(r))\right) .
$$

Notice that

$$
H(J(r))=\operatorname{diag}\left(A_{1}^{2}, \ldots, A_{n}^{2}\right), \quad \partial_{i} J(r)=A_{i} \partial_{\bar{i}} r
$$


one has

$$
\begin{aligned}
& b_{0}(z) r(z) \\
& \quad=\frac{-r(z)}{2 n(n+1) J(r)} \operatorname{tr}\left(\left[I_{n}-\frac{(\partial r) \otimes(\bar{\partial} r)}{J(r)}\right]\left[H(J(r))-\frac{(\partial J(r)) \otimes(\bar{\partial} J(r))}{J(r)}\right]\right) \\
& \quad=\frac{-r(z)}{2 n(n+1) J(r)}\left(\|A\|^{2}-2 \frac{\sum_{j=1}^{n} A_{j}^{2}\left|\partial_{j} r(z)\right|^{2}}{J(r)}+\frac{\left|\sum_{j=1}^{n} A_{j}\left(\partial_{j} r\right)^{2}\right|^{2}}{J(r)^{2}}\right) .
\end{aligned}
$$

Therefore,

$$
b_{0}(z)=\frac{-1}{2 n(n+1) J(r)}\left(\|A\|^{2}-2 \frac{\sum_{j=1}^{n} A_{j}^{2}\left|\partial_{j} r(z)\right|^{2}}{J(r)}+\frac{\left|\sum_{j=1}^{n} A_{j}\left(\partial_{j} r\right)^{2}\right|^{2}}{J(r)^{2}}\right) .
$$

By Theorem 2.1, one can easily see that if $\rho(z)=-\mathrm{e}^{-u(z)}$ with $u$ is the potential function Kähler-Einstein metric then

$$
\operatorname{det} H(\rho)=\operatorname{det} H\left(\rho^{1}\right) \quad \text { on } \partial D(A) \text {, }
$$

since $\rho(z)=\rho^{1}(z)+A_{3}(z)$ with $A_{3}(z)=O\left(\delta(z)^{3}\right)$ on $D(A)$ and $H\left(A_{3}\right)(z)=0$ on $\partial D(A)$.

In order to calculate $\operatorname{det} H\left(\rho^{1}\right)$ on $\partial D(A)$, we need the following lemma.

Lemma 3.1. Let $A=\left(A_{1}, \ldots, A_{n}\right)$ and $B=\left(B_{1}, \ldots, B_{n}\right)$ be two row vectors in $\mathbb{C}^{n}$. Then

$$
\operatorname{det}\left(I_{n}-A^{*} B-B^{*} A\right)=|1-\langle A, B\rangle|^{2}-|A|^{2}|B|^{2} .
$$

Moreover, if $\langle B, A\rangle \neq 1$ then

$$
\left(I_{n}-A^{*} B\right)^{-1}=I_{n}+\frac{1}{1-\langle B, A\rangle} A^{*} B .
$$

Proof. First, we consider the case $A=|A|(0, \ldots, 1)=|A| e_{n}$. Then

$$
\begin{aligned}
\operatorname{det}\left(I_{n}-A^{*} B-B^{*} A\right) & =1-|A|\left(B_{n}+\bar{B}_{n}\right)-|A|^{2} \sum_{j=1}^{n-1}\left|B_{j}\right|^{2} \\
& =1-|A|\left(B_{n}+\bar{B}_{n}\right)+|A|^{2}\left|B_{n}\right|^{2}-|A|^{2}|B|^{2} \\
& =|1-\langle A, B\rangle|^{2}-|A|^{2}|B|^{2}
\end{aligned}
$$


For a general row vector $A$, we choose a unitary matrix $U=\left[\alpha_{i j}\right]$ so that $A U=|A| e_{n}$. Then

$$
\begin{aligned}
\operatorname{det}\left(I_{n}-A^{*} B-B^{*} A\right) & =\operatorname{det}\left(I_{n}-(A U)^{*}(B U)-(B U)^{*}(A U)\right) \\
& =|1-\langle A U, B U\rangle|^{2}-|A U|^{2}|B U|^{2} \\
& =|1-\langle A, B\rangle|^{2}-|A|^{2}|B|^{2}
\end{aligned}
$$

Since

$$
\begin{aligned}
\left(I_{n}-A^{*} B\right)^{-1} & =I_{n}+\sum_{j=1}^{\infty}\left(A^{*} B\right)^{j}=I_{n}+\sum_{j=1}^{\infty}\left(\langle B, A\rangle^{j-1} A^{*} B\right. \\
& =I_{n}+\frac{1}{1-\langle B, A\rangle} A^{*} B .
\end{aligned}
$$

So, the proof of the lemma is complete.

The main purpose of this section is to prove the following theorem.

Theorem 3.1. Let $u$ be the potential function for the Kähler-Einstein metric on $D(A)$, and let $\rho(z)=-\mathrm{e}^{-u(z)}$ on $D$. Then $\rho(z)$ is strictly plurisubharmonic in $D(A)$.

Proof. We first prove $\operatorname{det} H(\rho) \geq 0$ on $\partial D(A)$. By (3.9), it suffices to prove

$$
\operatorname{det} H\left(\rho^{1}\right) \geq 0 \quad \text { on } \partial D(A) \text {. }
$$

Let

$$
B=(n+1) J(r) b_{0} \bar{\partial} r+\bar{\partial} J(r)
$$

be the row vector. Notice

$$
|\partial J|^{2}=\sum_{j=1}^{n} A_{j}^{2}\left|\partial_{j} r(z)\right|^{2}, \quad \bar{R} J(r)=\sum_{j=1}^{n} A_{j}\left(\partial_{j} r(z)\right)^{2}
$$

and $\operatorname{det} H\left(a+b r(z)^{k}\right)=\operatorname{det} H(a)$ on $\partial D(A)$ for any $k \geq 3$. Moreover, by the fact that $|\partial r|^{2}=J(r)$ on $\partial D(A)$ and Lemma 3.1, for any $z \in \partial D(A)$, one has

$\operatorname{det} H\left(\rho^{1}\right) J(r)^{n /(n+1)}$

$$
=J(r)^{n /(n+1)} \operatorname{det} H\left(\frac{r-b_{0} r^{2}}{J(r)^{1 /(n+1)}}\right)
$$




$$
\begin{aligned}
& =\operatorname{det}\left[H(r)-2 b_{0}(\partial r) \otimes(\bar{\partial} r)-\frac{((\partial J) \otimes(\bar{\partial} r)+(\partial r) \otimes(\bar{\partial} J))}{(n+1) J(r)}\right] \\
& =\operatorname{det}\left[I_{n}-\frac{(B \otimes(\partial r)+(\partial r) \otimes B}{(n+1) J(r)}\right] \\
& =\left|1-\frac{\langle B, \bar{\partial} r\rangle}{(n+1) J(r)}\right|^{2}-\frac{\|B\|^{2} \|\left.\partial r\right|^{2}}{(n+1)^{2} J(r)^{2}} \\
& =\left|1-\frac{(n+1) b_{0} J(r)|\partial r|^{2}+\bar{R} J}{(n+1) J(r)}\right|^{2} \\
& -\frac{(n+1)^{2} J(r)^{2} b_{0}^{2}|\partial r|^{2}+2(n+1) J(r) b_{0} \operatorname{Re}(\bar{R} J)+|\partial J(r)|^{2}}{(n+1)^{2} J(r)} \\
& =1-2 b_{0}|\partial r|^{2}-\frac{2 \operatorname{Re}(\bar{R} J)}{(n+1) J(r)}+b_{0}^{2}|\partial r|^{4}+2 \frac{b_{0}|\partial r|^{2}}{(n+1) J(r)} \operatorname{Re}(\bar{R} J(r)) \\
& +\frac{|\bar{R} J(r)|^{2}}{(n+1)^{2} J(r)^{2}}-b_{0}(z)^{2} J(r)|\partial r|^{2}-\frac{2 b_{0}}{(n+1)} \operatorname{Re}(\bar{R} J(r))-\frac{|\partial J(r)|^{2}}{(n+1)^{2} J(r)} \\
& =1-2 b_{0}|\partial r|^{2}-\frac{2 \operatorname{Re}(\bar{R} J)}{(n+1) J(r)}+\frac{|\bar{R} J(r)|^{2}}{(n+1)^{2} J(r)^{2}}-\frac{|\partial J(r)|^{2}}{(n+1)^{2} J(r)} \\
& \geq\left(1-\frac{|\bar{R} J(r)|}{(n+1) J(r)}\right)^{2}-\frac{A_{n}^{2}}{(n+1)^{2}}-2 b_{0} J(r) \\
& \geq\left(1-\frac{|\bar{R} J(r)|}{(n+1) J(r)}\right)^{2}-\frac{A_{n}^{2}}{(n+1)^{2}} \\
& +\frac{\|A\|^{2}-2 \sum_{j=1}^{n} A_{j}^{2}\left|\partial_{j} r\right|^{2} J(r)^{-1}+\left|\sum_{j=1}^{n} A_{j}\left(\partial_{j} r\right)^{2}\right|^{2} J(r)^{-2}}{n(n+1)} \\
& \geq\left(1-\frac{A_{n}}{(n+1)}\right)^{2}-\frac{A_{n}^{2}}{(n+1)^{2}} \\
& +\frac{\|A\|^{2}-2 A_{n}^{2}+\left|\sum_{j=1}^{n} A_{j}\left(\partial_{j} r\right)^{2}\right|^{2} J(r)^{-2}}{n(n+1)}
\end{aligned}
$$




$$
\begin{aligned}
& \geq 1-\frac{2}{n+1} A_{n}-\frac{A_{n}^{2}}{n(n+1)}+\frac{\left|\sum_{j=1}^{n} A_{j}\left(\partial_{j} r\right)^{2}\right|^{2} J(r)^{-2}}{n(n+1)} \\
& >0
\end{aligned}
$$

if $n \geq 2$ (in the above inequalities, we use the assumption: $0 \leq A_{1} \leq \cdots \leq$ $\left.A_{n}\right)$. When $n=1$, we pick up the term $\left|\sum_{j=1}^{n} A_{j}\left(\partial_{j} r\right)^{2}\right|^{2} J(r)^{-2}=A_{n}^{2}$ in the estimation of $\operatorname{det} H\left(\rho^{1}\right)$ on $\partial D(A)$; we have

$$
\operatorname{det} H(\rho) \geq 1-\frac{2 A_{n}}{n+1}=1-A_{n}>0 \quad \text { on } \partial D(A)
$$

Notice that $J(\rho)=1$ on $D(A)$, then

$$
\operatorname{det} H(\rho)=\frac{\operatorname{det} H(\rho)}{J(\rho)}=\mathrm{e}^{u(z)}\left(1-|\partial u|_{u}^{2}\right) .
$$

It was proved by the author in [14] that $\operatorname{det} H(\rho)$ attains its minimum over $\overline{D(A)}$ at the some point on $\partial D(A)$. By the first step, we have $\operatorname{det} H(\rho)>0$ on $\partial D(A)$. Therefore, $\operatorname{det} H(\rho)>0$ on $D(A)$ and the proof of the theorem is complete.

As a corollary of Theorems 3.2 and 1.1, we have proved the following result.

Corollary 3.1. If $g$ is the Kähler-Einstein metric on $D(A)$ then $\lambda_{1}$ $\left(\Delta_{g}\right)=n^{2}$.

This gives the proof of part (i) of Theorem 1.3.

\section{Holomorphic bisectional curvatures}

If $(M, g)$ is a Kähler manifold with Kähler metric $g$, then it is well known (see [20]) that the curvature tensor is given by the following formula:

$$
R_{i \bar{j} k \bar{\ell}}=-\frac{\partial^{2} g_{i \bar{j}}}{\partial z_{k} \partial \bar{z}_{\ell}}+g^{p \bar{q}} \frac{\partial g_{i \bar{q}}}{\partial z_{k}} \frac{\partial g_{p \bar{j}}}{\partial \bar{z}_{\ell}} .
$$

Assumes that the Kähler metric $g$ is induced by a strictly plurisubharmonic potential function $u$ on $M$ with $u=+\infty$ on $\partial M$ :

$$
g_{i \bar{j}}=\frac{\partial^{2} u}{\partial z_{i} \partial \bar{z}_{j}}=u_{i \bar{j}}, \quad \rho(z)=-e^{-u(z)},
$$


where $\rho$ becomes a negative defining function for $M$. We first provide a formula for the curvature tensor with the components of holomorphic bisectional curvature plus some derivatives of the defining function $\rho$.

Proposition 4.1. Let $u=-\log (-\rho)$ be strictly plurisubharmonic in $D$. Then

$$
R_{i \bar{j} k \bar{\ell}}=-\left(u_{i \bar{j}} u_{k \bar{\ell}}+u_{k \bar{j}} u_{i \bar{\ell}}\right)+E_{i \bar{j} k \bar{\ell}}
$$

where

$$
\begin{aligned}
E_{i \bar{j} k \bar{\ell}}= & -\frac{\rho_{i \bar{j} k \bar{\ell}}}{-\rho}+\frac{1}{\rho^{2}} g^{p \bar{q}}\left[\rho_{i \bar{q} k} \rho_{p \bar{j} \bar{\ell}}+\rho_{i \bar{q} k} u_{p} \rho_{\bar{j} \bar{\ell}}+\rho_{p \bar{j} \bar{\ell}} u_{\bar{q}} \rho_{i k}\right] \\
& -\left(1-|\partial u|_{g}^{2}\right) \frac{\rho_{i k} \rho_{\bar{j} \bar{\ell}}}{\rho^{2}} .
\end{aligned}
$$

Note: A theorem of Lu Qi-Keng [17] states that if $g$ is a Bergman metric for a bounded domain in $D$ in $\mathbb{C}^{n}$ then $D$ is biholomorphic to the unit ball in $\mathbb{C}^{n}$ if and only if $R_{i \bar{j} k \bar{\ell}}=-\left(u_{i \bar{j}} u_{k \bar{\ell}}+u_{k \bar{j}} u_{i \bar{\ell}}\right)$ on $D$ (or $E_{i \bar{j} k \bar{\ell}}=0$ on $\left.D\right)$. Next, we prove Proposition 4.1.

Proof. Notice that

$$
\begin{aligned}
u & =-\log (-\rho), \quad u_{i}=\frac{\rho_{i}}{-\rho}, u_{i \bar{j}}=\frac{\rho_{i \bar{j}}}{-\rho}+u_{i} u_{\bar{j}}, u_{i k}=\frac{\rho_{i k}}{-\rho}+u_{i} u_{k} \\
u_{i \bar{j} k} & =\frac{\rho_{i \bar{j} k}}{-\rho}+\frac{\rho_{i \bar{j}}}{-\rho} u_{k}+u_{i k} u_{\bar{j}}+u_{i} u_{k \bar{j}} \\
& =\frac{\rho_{i \bar{j} k}}{-\rho}+\frac{\rho_{i \bar{j}}}{-\rho} u_{k}+\frac{\rho_{i k}}{-\rho} u_{\bar{j}}+\frac{\rho_{k \bar{j}}}{-\rho} u_{i}+2 u_{i} u_{k} u_{\bar{j}}
\end{aligned}
$$

and

$$
\begin{aligned}
u_{i \bar{j} k \bar{\ell}}= & \frac{\rho_{i \bar{j} k \bar{\ell}}}{-\rho}+\frac{\rho_{i \bar{j} k}}{-\rho} u_{\bar{\ell}}+\frac{\rho_{i \bar{j} \bar{\ell}}}{-\rho} u_{k}+\frac{\rho_{i \bar{j}}}{-\rho} u_{k} u_{\bar{\ell}}+\frac{\rho_{i \bar{j}}}{-\rho} u_{k \bar{\ell}} \\
& +\frac{\rho_{i k \bar{\ell}}}{-\rho} u_{\bar{j}}+\frac{\rho_{i k}}{-\rho} u_{\bar{j}} u_{\bar{\ell}}+\frac{\rho_{i k}}{-\rho} u_{\bar{j} \bar{\ell}}+\frac{\rho_{k \bar{j} \bar{\ell}}}{-\rho} u_{i}+\frac{\rho_{k \bar{j}}}{-\rho} u_{i} u_{\bar{\ell}}+\frac{\rho_{k \bar{j}}}{-\rho} u_{i \bar{\ell}} \\
& +2 u_{i \bar{\ell}} u_{k} u_{\bar{j}}+2 u_{i} u_{k \bar{\ell}} u_{\bar{j}}+2 u_{i} u_{k} u_{\bar{j} \bar{\ell}} \\
= & \frac{\rho_{i \bar{j} k \bar{\ell}}}{-\rho}+\frac{\rho_{i \bar{j} k}}{-\rho} u_{\bar{\ell}}+\frac{\rho_{i \bar{j} \bar{\ell}}}{-\rho} u_{k}+\frac{\rho_{i k \bar{\ell}}}{-\rho} u_{\bar{j}}+\frac{\rho_{k \bar{j} \bar{\ell}}}{-\rho} u_{i} \\
& +u_{i \bar{j}}\left(u_{k} u_{\bar{\ell}}+u_{k \bar{\ell}}\right)+u_{i k}\left(u_{\bar{j}} u_{\bar{\ell}}+u_{\bar{j} \bar{\ell}}\right)+u_{k \bar{j}}\left(u_{i} u_{\bar{\ell}}+u_{i \bar{\ell}}\right) \\
& -3 u_{i} u_{\bar{j}} u_{k} u_{\bar{\ell}}+u_{i \bar{\ell}} u_{k} u_{\bar{j}}+u_{i} u_{k \bar{\ell}} u_{\bar{j}}+u_{i} u_{k} u_{\bar{j} \bar{\ell}} .
\end{aligned}
$$


By (4.5) and (4.6), one has

$$
u_{i \bar{q} k}=\frac{\rho_{i \bar{q} k}}{-\rho}+u_{i \bar{q}} u_{k}-u_{i} u_{\bar{q}} u_{k}+u_{i k} u_{\bar{q}}+u_{i} u_{k \bar{q}}
$$

and

$$
u_{p \bar{j} \bar{\ell}}=\frac{\rho_{p \bar{j} \bar{\ell}}}{-\rho}+u_{p \bar{j}} u_{\bar{\ell}}-u_{p} u_{\bar{j}} u_{\bar{\ell}}+u_{p \bar{\ell}} u_{\bar{j}}+u_{p} u_{\bar{j} \bar{\ell}}
$$

Thus,

$$
\begin{aligned}
& g^{p \bar{q}} u_{i \bar{q} k} u_{p \bar{j} \bar{\ell}} \\
& =\left(g^{p \bar{q}} \frac{\rho_{i \bar{q} k}}{(-\rho)}+\delta_{i p} u_{k}-g^{p \bar{q}} u_{i} u_{\bar{q}} u_{k}+u_{i k} g^{p \bar{q}} u_{\bar{q}}+u_{i} \delta_{k p}\right) \\
& \times\left(\frac{\rho_{p \bar{j} \bar{\ell}}}{(-\rho)}+u_{p \bar{j}} u_{\bar{\ell}}-u_{p} u_{\bar{j}} u_{\bar{\ell}}+u_{p \bar{\ell}} u_{\bar{j}}+u_{p} u_{\bar{j} \bar{\ell}}\right) \\
& =g^{p \bar{q}} \frac{\rho_{i \bar{q} k}}{(-\rho)} \frac{\rho_{p \bar{j} \bar{\ell}}}{(-\rho)}+g^{p \bar{q}} \frac{\rho_{i \bar{q} k}}{(-\rho)}\left(u_{p \bar{j}} u_{\bar{\ell}}-u_{p} u_{\bar{j}} u_{\bar{\ell}}+u_{p \bar{\ell}} u_{\bar{j}}+u_{p} u_{\bar{j} \bar{\ell}}\right) \\
& +\frac{\rho_{p \bar{j} \bar{\ell}}}{(-\rho)}\left(\delta_{i p} u_{k}-g^{p \bar{q}} u_{i} u_{\bar{q}} u_{k}+u_{i k} g^{p \bar{q}} u_{\bar{q}}+u_{i} \delta_{k p}\right) \\
& +u_{i \bar{j}} u_{k} u_{\bar{\ell}}-u_{i} u_{k} u_{\bar{j}} u_{\bar{\ell}}+u_{i \bar{\ell}} u_{k} u_{\bar{j}}+u_{i} u_{k} u_{\bar{j} \bar{\ell}} \\
& -u_{i} u_{\bar{j}} u_{k} u_{\bar{\ell}}+|\partial u|_{g}^{2} u_{i} u_{\bar{j}} u_{k} u_{\bar{\ell}}-u_{i} u_{\bar{j}} u_{k} u_{\bar{\ell}}-|\partial u|_{g}^{2} u_{i} u_{k} u_{\bar{j} \bar{\ell}} \\
& +u_{i k} u_{j}^{-} u_{\bar{\ell}}-|\partial u|_{g}^{2} u_{i k} u_{\bar{j}}^{-} u_{\bar{\ell}}+u_{i k} u_{\bar{j}} u_{\bar{\ell}}+|\partial u|_{g}^{2} u_{i k} u_{\bar{j} \bar{\ell}} \\
& +u_{k \bar{j}} u_{i} u_{\bar{\ell}}-u_{i} u_{\bar{\ell}} u_{k} u_{\bar{j}}+u_{i} u_{\bar{j}} u_{k \bar{\ell}}+u_{i} u_{k} u_{\bar{j} \bar{\ell}} \\
& =g^{p \bar{q}} \frac{\rho_{i \bar{q} k}}{(-\rho)} \frac{\rho_{p \bar{j} \bar{\ell}}}{(-\rho)}+g^{p \bar{q}} \frac{\rho_{i \bar{q} k}}{(-\rho)}\left(-u_{p} u_{\bar{j}} u_{\bar{\ell}}+u_{p} u_{\bar{j} \bar{\ell}}\right)+\frac{\rho_{i \bar{j} k}}{-\rho} u_{\bar{\ell}}+\frac{\rho_{i k \bar{\ell}}}{-\rho} u_{\bar{j}} \\
& +\frac{\rho_{i \bar{j} \bar{\ell}}}{(-\rho)} u_{k}+\frac{\rho_{k \bar{j} \bar{\ell}}}{-\rho} u_{i}+\frac{\rho_{p \bar{j} \bar{\ell}}}{-\rho}\left(-g^{p \bar{q}} u_{i} u_{\bar{q}} u_{k}+u_{i k} g^{p \bar{q}} u_{\bar{q}}\right) \\
& +u_{i \bar{j}} u_{k} u_{\bar{\ell}}+u_{i \bar{\ell}} u_{k} u_{\bar{j}}+u_{i} u_{k} u_{\bar{j} \bar{\ell}}+u_{i k} u_{\bar{j}} u_{\bar{\ell}}+u_{k \bar{j}} u_{i} u_{\bar{\ell}}+u_{i} u_{\bar{j}} u_{k \bar{\ell}} \\
& -3 u_{i} u_{k} u_{\bar{j}} u_{\bar{\ell}}-\left(1-|\partial u|_{g}^{2}\right) u_{i} u_{\bar{j}} u_{k} u_{\bar{\ell}} \\
& +\left(1-|\partial u|_{g}^{2}\right) u_{i k} u_{\bar{j}} u_{\bar{\ell}}+|\partial u|_{g}^{2} u_{i k} u_{\bar{j} \bar{\ell}}+\left(1-|\partial u|_{g}^{2}\right) u_{i} u_{k} u_{\bar{j} \bar{\ell}} .
\end{aligned}
$$

Therefore, by (4.1), (4.7), (4.8) and (4.5)

$$
\begin{aligned}
R_{i \bar{j} k \bar{\ell}}= & -\left(u_{i \bar{j}} u_{k \bar{\ell}}+u_{k \bar{j}} u_{i \bar{\ell}}\right)-\frac{\rho_{i \bar{j} k \bar{\ell}}}{-\rho}+g^{p \bar{q}} \frac{\rho_{i \bar{q} k}}{-\rho} \frac{\rho_{p \bar{j} \bar{\ell}}}{-\rho} \\
& +\frac{\rho_{i \bar{q} k}}{-\rho} g^{p \bar{q}} u_{p}\left(-u_{\bar{j}} u_{\bar{\ell}}+u_{\bar{j} \bar{\ell}}\right)+\frac{\rho_{p \bar{j} \bar{\ell}}}{-\rho} g^{p \bar{q}} u_{\bar{q}}\left(-u_{i} u_{\bar{q}} u_{k}+u_{i k}\right) \\
& -\left(1-|\partial u|_{g}^{2}\right)\left(u_{i k} u_{\bar{j} \bar{\ell}}+u_{i} u_{\bar{j}} u_{k} u_{\bar{\ell}}-u_{i} u_{k} u_{\bar{j} \bar{\ell}}-u_{i k} u_{\bar{j}} u_{\bar{\ell}}\right)
\end{aligned}
$$




$$
\begin{aligned}
= & -\left(u_{i \bar{j}} u_{k \bar{\ell}}+u_{k \bar{j}} u_{i \bar{\ell})}\right)-\frac{\rho_{i \bar{j} k \bar{\ell}}}{-\rho} \\
& +\frac{1}{\rho^{2}} g^{p \bar{q}}\left[\rho_{i \bar{q} k} \rho_{p \bar{j} \bar{\ell}}+\rho_{i \bar{q} k} u_{p} \rho_{\bar{j} \bar{\ell}}+\rho_{p \bar{j} \bar{\ell}} u_{\bar{q}} \rho_{i k}\right]-\left(1-|\partial u|_{g}^{2}\right) \frac{\rho_{i k} \rho_{\bar{j} \bar{\ell}}}{\rho^{2}} .
\end{aligned}
$$

This completes the proof of the proposition.

Next we construct a Kähler metric $g$ on $D(A)$ so that its holomorphic bisectional curvature $\mathcal{K}_{g} \geq-1$ and $\lambda_{1}\left(\Delta_{g}\right)=n^{2}$. On $D(A)$, we let

$$
\rho(z)=2 r(z)-\frac{\alpha}{2} r(z)^{2}, \quad 1 \leq \alpha \leq 2,
$$

where $r(z)$ is given by (3.2). Notice

$$
H(r)=I_{n}, \quad H(\rho)=(2-\alpha r(z)) I_{n}-\alpha(\partial r) \otimes(\bar{\partial} r) .
$$

It is easy to show that $r(z)$ and $-\log (-\rho(z))$ both are strictly plurisubharmonic negative defining functions for $D(A)$. Let

$$
u(z)=-\log (-\rho), \quad g=g_{i \bar{j}} d z_{i} \otimes d \bar{z}_{j}=\frac{\partial^{2} u}{\partial z_{i} \partial \bar{z}_{j}} d z_{i} \otimes d \bar{z}_{j}
$$

is a Kähler metric on $D(A)$ induced by $u$. It is easy to prove that $(D(A), g)$ is a complete Kähler manifold. We will prove the following theorem.

Theorem 4.1. Let $g$ be a Kähler metric defined by (4.9) and (4.11) on $D(A)$. If $A_{n} \leq 2 / 5$, then the holomorphic bisectional curvature $\mathcal{K}_{g} \geq-1$ on $D(A)$.

Proof. For simplicity, we write

$$
\rho(z)=2 r(z)-\frac{\alpha}{2} r(z)^{2}=: \phi(r(z)) .
$$

Then

$$
\begin{gathered}
\rho_{i}(z)=\phi^{\prime}(r) r_{i}, \quad \rho_{i \bar{j}}=\phi^{\prime}(r) r_{i \bar{j}}-\alpha r_{i} r_{\bar{j}}, \quad \rho_{i k}=\phi^{\prime}(r) r_{i k}-\alpha r_{i} r_{k} \\
\rho_{i k \bar{q}}=-\alpha\left[r_{\bar{q}} r_{i k}+r_{i \bar{q}} r_{k}+r_{i} r_{k \bar{q}}\right]
\end{gathered}
$$


and

$$
\begin{aligned}
\rho_{i \bar{j} k \bar{\ell}} & =-\alpha \frac{\partial}{\partial \bar{z}_{\ell}}\left[r_{k} r_{i \bar{j}}+r_{i k} r_{\bar{j}}+r_{i} r_{k \bar{j}}\right] \\
& =-\alpha\left[r_{k \bar{\ell}} r_{i \bar{j}}+r_{i k} r_{\bar{j} \bar{\ell}}+r_{i \bar{\ell}} r_{k \bar{j}}\right] \\
& =-\alpha\left(\delta_{i j} \delta_{k \ell}+A_{i} A_{j} \delta_{i k} \delta_{j \ell}+\delta_{i \ell} \delta_{k j}\right) .
\end{aligned}
$$

From (4.13), one has

$$
\rho_{i \bar{j}}=\phi^{\prime}(r)\left(\delta_{i j}-\frac{\alpha}{\phi^{\prime}(r)} r_{i} r_{\bar{j}}\right), \quad \rho^{i \bar{j}}=\frac{1}{\phi^{\prime}(r)}\left[\delta_{i j}+\frac{\alpha}{\phi^{\prime}(r)-\alpha|\partial r|^{2}} r_{\bar{i}} r_{j}\right] .
$$

From (4.11) and (4.13), one has

$$
\begin{aligned}
g_{i \bar{j}} & =\frac{1}{-\rho}\left[\rho_{i \bar{j}}+\frac{1}{-\rho} \rho_{i} \rho_{\bar{j}}\right] \\
& =\frac{1}{-\rho}\left[\phi^{\prime}(r) \delta_{i j}-\alpha r_{i} r_{\bar{j}}+\frac{\phi^{\prime}(r)^{2}}{-\rho} r_{i} r_{\bar{j}}\right] \\
& =\frac{\phi^{\prime}(r)}{-\rho}\left[\delta_{i j}+\frac{\phi^{\prime}(r)^{2}+\alpha \rho}{\phi^{\prime}(r)(-\rho)} r_{i} r_{\bar{j}}\right]
\end{aligned}
$$

and

(4.18) $g^{i \bar{j}}=\frac{-\rho}{\phi^{\prime}(r)}\left[\delta_{i j}-b r_{i} r_{j}\right] \quad$ and $b=\frac{\phi^{\prime}(r)^{2}+\alpha \rho}{\phi^{\prime}(r)(-\rho)+\left(\phi^{\prime}(r)^{2}+\alpha \rho\right)|\partial r|^{2}}$.

Therefore,

$$
\begin{aligned}
\sum_{q=1}^{n} g^{p \bar{q}} \rho_{i k \bar{q}} & =\frac{-\rho}{\phi^{\prime}(r)}\left[\delta_{p q}-b r_{\bar{p}} r_{q}\right]\left(-\alpha\left[r_{\bar{q}} r_{i k}+r_{i \bar{q}} r_{k}+r_{i} r_{k \bar{q}}\right]\right) \\
& =-\alpha \frac{-\rho}{\phi^{\prime}(r)}\left(r_{\bar{p}} r_{i k}+r_{i \bar{p}} r_{k}+r_{i} r_{k \bar{p}}-b r_{\bar{p}}\left(|\partial r|^{2} r_{i k}+2 r_{i} r_{k}\right)\right)
\end{aligned}
$$

Hence,

$$
\begin{aligned}
& \sum_{p, q=1}^{n} g^{p \bar{q}} \rho_{i k \bar{q}} u_{p} \\
& \quad=-\alpha \frac{-\rho}{\phi^{\prime}(r)}\left(r_{\bar{p}} r_{i k}+r_{i \bar{p}} r_{k}+r_{i} r_{k \bar{p}}-b r_{\bar{p}}\left(|\partial r|^{2} r_{i k}+2 r_{i} r_{k}\right) \frac{\phi^{\prime}(r)}{-\rho} r_{p}\right. \\
& \quad=-\alpha\left(1-b|\partial r|^{2}\right)\left(|\partial r|^{2} r_{i k}+2 r_{i} r_{k}\right) .
\end{aligned}
$$




$$
\frac{1}{\rho^{2}} \sum_{p, q=1}^{n} g^{p \bar{q}} u_{p} \rho_{i k \bar{q}} \rho_{\bar{j} \bar{\ell}}=-\alpha \frac{\left(1-b|\partial r|^{2}\right)}{\rho^{2}}\left(|\partial r|^{2} r_{i k}+2 r_{i} r_{k}\right)\left[\phi^{\prime}(r) r_{\bar{j} \bar{\ell}}-\alpha r_{\bar{j}} r_{\bar{\ell}}\right]
$$

and

$$
\frac{1}{\rho^{2}} \sum_{p, q=1}^{n} g^{p \bar{q}} u_{\bar{q}} \rho_{p \bar{j} \bar{\ell}} \rho_{i k}=-\alpha \frac{\left(1-b|\partial r|^{2}\right)}{\rho^{2}}\left[|\partial r|^{2} r_{\bar{j} \bar{\ell}}+2 r_{\bar{j}} r_{\bar{\ell}}\right]\left[\phi^{\prime}(r) r_{i k}-\alpha r_{i} r_{k}\right] .
$$

Using

$$
\begin{aligned}
\left(|\partial r|^{2} r_{i k}+2 r_{i} r_{k}\right)\left[\phi^{\prime}(r) r_{\bar{j} \bar{\ell}}-\alpha r_{\bar{j}} r_{\bar{\ell}}\right]+\left[|\partial r|^{2} r_{\bar{j} \bar{\ell}}+2 r_{\bar{j}} r_{\bar{\ell}}\right]\left[\phi^{\prime}(r) r_{i k}-\alpha r_{i} r_{k}\right] \\
=-4 \alpha r_{i} r_{\bar{j}} r_{k} r_{\bar{\ell}}+2 \phi^{\prime}(r)|\partial r|^{2} r_{i k} r_{\bar{j} \bar{\ell}}+2 \phi^{\prime}(r)\left(r_{\bar{j} \bar{\ell}} r_{i} r_{k}+r_{i k} r_{\bar{j}} r_{\bar{\ell}}\right) \\
\quad-\alpha|\partial r|^{2}\left(r_{i k} r_{\bar{j}} r_{\bar{\ell}}+r_{\bar{j} \bar{\ell}} r_{i} r_{k}\right),
\end{aligned}
$$

(4.21), (4.22) and (4.23), one has

$$
\begin{aligned}
\frac{1}{\rho^{2}} \sum_{p, q=1}^{n} g^{p \bar{q}}\left[u_{p} \rho_{i k \bar{q}} \rho_{\bar{j} \bar{\ell}}+u_{\bar{q}} \rho_{p \bar{j} \bar{\ell}} \rho_{i k}\right] \\
=-2 \alpha \frac{\left(1-b|\partial r|^{2}\right)}{\rho^{2}}\left[\phi^{\prime}(r)|\partial r|^{2} r_{i k} r_{\bar{j} \bar{\ell}}+\left(\phi^{\prime}(r)-\frac{\alpha}{2}|\partial r|^{2}\right)\right. \\
\left.\quad \times\left(r_{i k} r_{\bar{j}} r_{\bar{\ell}}+r_{\bar{j} \bar{\ell}} r_{i} r_{k}\right)-2 \alpha r_{i} r_{\bar{j}} r_{k} r_{\bar{\ell}}\right] \\
=-2 \alpha \frac{\left(1-b|\partial r|^{2}\right)}{\rho^{2}}\left[\phi^{\prime}|\partial r|^{2} A_{i} \delta_{i k} A_{j} \delta_{j \ell}+\left(\phi^{\prime}-\frac{\alpha}{2}|\partial r|^{2}\right)\right. \\
\left.\quad \times\left(A_{i} \delta_{i k} r_{\bar{j}} r_{\bar{\ell}}+A_{j} \delta_{j \ell} r_{i} r_{k}\right)-2 \alpha r_{i} r_{\bar{j}} r_{k} r_{\bar{\ell}}\right] .
\end{aligned}
$$

Since

$$
|\partial u|_{g}^{2}=\sum_{p, q=1}^{n} g^{p \bar{q}} u_{p} u_{\bar{q}}=\frac{-\rho}{\phi^{\prime}(r)}\left(\delta_{p q}-b r_{\bar{p}} r_{q}\right) \frac{\phi^{\prime}(r)^{2}}{\rho^{2}} r_{p} r_{\bar{q}}=\frac{\phi^{\prime}(r)}{-\rho}|\partial r|^{2}\left(1-b|\partial r|^{2}\right),
$$

one has

$$
\begin{aligned}
1-b|\partial r|^{2} & =1-\frac{\left(\phi^{\prime}(r)^{2}+\alpha \rho\right)|\partial r|^{2}}{\phi^{\prime}(r)(-\rho)+\left(\phi^{\prime}(r)^{2}+\alpha \rho\right)|\partial r|^{2}} \\
& =\frac{\phi^{\prime}(r)(-\rho)}{\phi^{\prime}(r)(-\rho)+\left(\phi^{\prime}(r)^{2}+\alpha \rho\right)|\partial r|^{2}}
\end{aligned}
$$




$$
=\frac{\phi^{\prime}(r)(-\rho) b}{\left(\phi^{\prime}(r)^{2}+\alpha \rho\right)}
$$

Thus,

$$
1-|\partial u|_{g}^{2}=1-\frac{\phi^{\prime}(r)^{2}|\partial r|^{2}}{\phi^{\prime}(r)(-\rho)+\left(\phi^{\prime}(r)^{2}+\alpha \rho\right)|\partial r|^{2}}=\frac{(-\rho)\left(\phi^{\prime}(r)-\alpha|\partial r|^{2}\right) b}{\left(\phi^{\prime}(r)^{2}+\alpha \rho\right)}
$$

and

$$
\begin{aligned}
\frac{1}{\rho^{2}}(1 & \left.-|\partial u|_{g}^{2}\right) \rho_{i k} \rho_{\bar{j} \bar{\ell}} \\
= & \frac{\left(\phi^{\prime}(r)-\alpha|\partial r|^{2}\right) b}{(-\rho)\left(\phi^{\prime}(r)^{2}+\alpha \rho\right)}\left(\phi^{\prime}(r) A_{i} \delta_{i k}-\alpha r_{i} r_{k}\right)\left(\phi^{\prime}(r) A_{j} \delta_{j \ell}-\alpha r_{\bar{j}} r_{\bar{\ell}}\right) \\
= & \frac{\left(\phi^{\prime}(r)-\alpha|\partial r|^{2}\right) b}{(-\rho)\left(\phi^{\prime}(r)^{2}+\alpha \rho\right)}\left(\phi^{\prime}(r)^{2} A_{j} A_{i} \delta_{i k} \delta_{j \ell}-\alpha \phi^{\prime}(r)\right. \\
& \left.\times\left(A_{j} \delta_{j \ell} r_{i} r_{k}+A_{i} \delta_{i k} r_{\bar{j}} r_{\bar{\ell}}\right)+\alpha^{2} r_{i} r_{\bar{j}} r_{k} r_{\bar{\ell}}\right)
\end{aligned}
$$

Therefore,

$$
\begin{aligned}
\frac{(-\rho)}{\rho^{2}} & \sum_{p, q=1}^{n} g^{p \bar{q}}\left[u_{p} \rho_{i k \bar{q}} \rho_{\bar{j} \bar{\ell}}+u_{\bar{q}} \rho_{p \bar{j} \bar{\ell}} \rho_{i k}\right]-\frac{(-\rho)}{\rho^{2}}\left(1-|\partial u|_{g}^{2}\right) \rho_{i k} \rho_{\bar{j} \bar{\ell}} \\
= & \frac{-2 \alpha \phi^{\prime}(r) b}{\left(\phi^{\prime}(r)^{2}+\alpha \rho\right)}\left[\phi^{\prime}|\partial r|^{2} A_{i} \delta_{i k} A_{j} \delta_{j \ell}+\left(\phi^{\prime}-\frac{\alpha}{2}|\partial r|^{2}\right)\right. \\
& \left.\times\left(A_{i} \delta_{i k} r_{\bar{j}} r_{\bar{\ell}}+A_{j} \delta_{j \ell} r_{i} r_{k}\right)-2 \alpha r_{i} r_{\bar{j}} r_{k} r_{\bar{\ell}}\right]-\frac{\left(\phi^{\prime}(r)-\alpha|\partial r|^{2}\right) b}{\left(\phi^{\prime}(r)^{2}+\alpha \rho\right)} \\
& \times\left(\phi^{\prime}(r)^{2} A_{j} A_{i} \delta_{i k} \delta_{j \ell}-\alpha \phi^{\prime}(r)\left(A_{j} \delta_{j \ell} r_{i} r_{k}+A_{i} \delta_{i k} r_{\bar{j}} r_{\bar{\ell}}\right)+\alpha^{2} r_{i} r_{\bar{j}} r_{k} r_{\bar{\ell}}\right) \\
= & -\frac{\left(\alpha|\partial r|^{2}+\phi^{\prime}(r)\right) \phi^{\prime}(r)^{2} b}{\left(\phi^{\prime}(r)^{2}+\alpha \rho\right)} A_{i} A_{j} \delta_{i k} \delta_{j \ell}-\frac{\alpha \phi^{\prime}(r)^{2} b}{\left(\phi^{\prime}(r)^{2}+\alpha \rho\right)} \\
& \times\left(A_{j} \delta_{j \ell} r_{i} r_{k}+A_{i} \delta_{i k} r_{\bar{j}} r_{\bar{\ell}}\right)+\frac{\alpha^{2} b\left(3 \phi^{\prime}(r)+\alpha|\partial r|^{2}\right)}{\left(\phi^{\prime}(r)^{2}+\alpha \rho\right)} r_{i} r_{\bar{j}} r_{k} r_{\bar{\ell}} .
\end{aligned}
$$

Therefore, by (4.4), (4.15) and (4.25), for any $\xi, \eta \in \mathbb{C}^{n}$, one has

$$
\begin{aligned}
& (-\rho) E_{i \bar{j} k \bar{\ell}} \xi_{i} \bar{\xi}_{j} \eta_{k} \bar{\eta}_{\ell} \\
& \geq \alpha\left(|\xi|^{2}|\eta|^{2}+\left|\sum_{i=1}^{n} A_{i} \xi_{i} \eta_{i}\right|^{2}+\left|\sum_{i=1}^{n} \xi_{i} \bar{\eta}_{i}\right|^{2}\right)
\end{aligned}
$$




$$
\begin{aligned}
& -\frac{\left(\alpha|\partial r|^{2}+\phi^{\prime}(r)\right) \phi^{\prime}(r)^{2} b}{\left(\phi^{\prime}(r)^{2}+\alpha \rho\right)}\left|\sum_{i=1}^{n} A_{i} \xi_{i} \eta_{i}\right|^{2} \\
& -\frac{2 \alpha \phi^{\prime}(r)^{2} b}{\left(\phi^{\prime}(r)^{2}+\alpha \rho\right)} \operatorname{Re}\left(\left(\sum_{j=1}^{n} A_{j} \bar{\xi}_{j} \bar{\eta}_{j}\right)\left(\sum_{i=1}^{n} \xi_{j} r_{i}\right)\left(\sum_{k=1}^{n} \eta_{k} r_{k}\right)\right) \\
& +\frac{\alpha^{2} b\left(3 \phi^{\prime}(r)+\alpha|\partial r|^{2}\right)}{\left(\phi^{\prime}(r)^{2}+\alpha \rho\right)}\left|\sum_{i=1}^{n} r_{i} \xi_{i}\right|^{2}\left|\sum_{k=1}^{n} r_{k} \eta_{k}\right|^{2} \\
& \geq \alpha\left(|\xi|^{2}|\eta|^{2}+\left|\sum_{i=1}^{n} A_{i} \xi_{i} \eta_{i}\right|^{2}+\left|\sum_{i=1}^{n} \xi_{i} \bar{\eta}_{i}\right|^{2}\right) \\
& -\frac{\left(\alpha|\partial r|^{2}+\phi^{\prime}(r)\right) \phi^{\prime}(r)^{2} b}{\left(\phi^{\prime}(r)^{2}+\alpha \rho\right)}\left|\sum_{i=1}^{n} A_{i} \xi_{i} \eta_{i}\right|^{2} \\
& -\frac{1}{4}\left[\frac{2 \alpha \phi^{\prime}(r)^{2} b}{\left(\phi^{\prime}(r)^{2}+\alpha \rho\right)}\right]^{2} \frac{\left(\phi^{\prime}(r)^{2}+\alpha \rho\right)}{\alpha^{2} b\left(3 \phi^{\prime}(r)+\alpha|\partial r|^{2}\right)}\left|\sum_{j=1}^{n} A_{j} \bar{\xi}_{j} \bar{\eta}_{j}\right|^{2} \\
& =\alpha\left(|\xi|^{2}|\eta|^{2}+\left|\sum_{i=1}^{n} \xi_{i} \bar{\eta}_{i}\right|^{2}\right)+\left[\alpha-\frac{\left(\alpha|\partial r|^{2}+\phi^{\prime}(r)\right) \phi^{\prime}(r)^{2} b}{\left(\phi^{\prime}(r)^{2}+\alpha \rho\right)}\right. \\
& \left.-\frac{\phi^{\prime}(r)^{4} b}{\left(\phi^{\prime}(r)^{2}+\alpha \rho\right)} \frac{1}{\left(3 \phi^{\prime}(r)+\alpha|\partial r|^{2}\right)}\right]\left|\sum_{j=1}^{n} A_{j} \bar{\xi}_{j} \bar{\eta}_{j}\right|^{2} \\
& =\alpha\left(|\xi|^{2}|\eta|^{2}+\left|\sum_{i=1}^{n} \xi_{i} \bar{\eta}_{i}\right|^{2}\right) \\
& +\alpha\left(1-\frac{\phi^{\prime}(r)^{2} b\left((4 / \alpha) \phi^{\prime}(r)^{2}+\alpha|\partial r|^{4}+4 \phi^{\prime}(r)|\partial r|^{2}\right)}{\left(\phi^{\prime}(r)^{2}+\alpha \rho\right)\left(3 \phi^{\prime}(r)+\alpha|\partial r|^{2}\right)}\right)\left|\sum_{j=1}^{n} A_{j} \bar{\xi}_{j} \bar{\eta}_{j}\right|^{2} \\
& =\alpha\left(|\xi|^{2}|\eta|^{2}+\left|\sum_{i=1}^{n} \xi_{i} \bar{\eta}_{i}\right|^{2}\right) \\
& +\alpha\left(1-\frac{\alpha \phi^{\prime}(r)^{2}\left(\phi^{\prime}(r)(2 / \alpha)+|\partial r|^{2}\right)^{2}}{\left(\phi^{\prime}(r)(-\rho)+\left(\phi^{\prime}(r)^{2}+\alpha \rho\right)|\partial r|^{2}\right)\left(3 \phi^{\prime}(r)+\alpha|\partial r|^{2}\right)}\right)\left|\sum_{j=1}^{n} A_{j} \bar{\xi}_{j} \bar{\eta}_{j}\right|^{2} .
\end{aligned}
$$

Notice

$$
16 \geq \phi^{\prime}(r)^{2} \geq \phi^{\prime}(r)^{2}+\alpha \rho=4-4 r(z)+2 r(z)^{2} \geq 2 \phi^{\prime}(r) \geq 4
$$

and since $|z|^{2}\left(1-A_{n}\right)<1$ on $D(A)$, one has

$$
\begin{aligned}
(4.28) \quad- & \rho(z)+2|\partial r(z)|^{2} \\
& \geq-2 r(z)+2|\partial r(z)|^{2}
\end{aligned}
$$




$$
\begin{aligned}
& \geq 2\left(1-|z|^{2}-\sum_{j=1}^{n} A_{j} \operatorname{Re} z_{j}^{2}+|z|^{2}+2 \sum_{j=1}^{n} A_{j} \operatorname{Re} z_{j}^{2}+\sum_{j=1}^{n} A_{j}^{2}\left|z_{j}\right|^{2}\right) \\
& =2\left(1+\sum_{j=1}^{n} A_{j} \operatorname{Re} z_{j}^{2}+\sum_{j=1}^{n} A_{j}^{2}\left|z_{j}\right|^{2}\right) \\
& \geq 2\left(1-\sum_{j=1}^{n} A_{j}^{2}\left|z_{j}\right|^{2}-\frac{1}{4}|z|^{2}+\sum_{j=1}^{n} A_{j}^{2}\left|z_{j}\right|^{2}\right) \\
& \geq 2\left(1-\frac{|z|^{2}}{4}\right) \\
& \geq 2\left(1-\frac{1}{4\left(1-A_{n}\right)}\right) \\
& =\frac{3-4 A_{n}}{2\left(1-A_{n}\right)}
\end{aligned}
$$

Therefore, with $A_{n}<3 / 4$

$$
\begin{aligned}
\phi^{\prime}(r)(-\rho)+\left(\phi^{\prime}(r)^{2}+\alpha \rho\right)|\partial r|^{2} & \geq \phi^{\prime}(r)(-\rho)+2 \phi^{\prime}(r)|\partial r|^{2} \\
& =\phi^{\prime}(r)\left[-\rho+2|\partial r|^{2}\right] \\
& \geq \frac{\left(3-4 A_{n}\right) \phi^{\prime}(r)}{2\left(1-A_{n}\right)} .
\end{aligned}
$$

Since $|\partial r(z)|^{2} \leq \phi^{\prime}(r)$ and computation in (4.29), one has $\phi^{\prime}(r)+|\partial r(z)|^{2}=$ $2-2 r(z)+|\partial r(z)|^{2} \leq 4$. Thus, since $\alpha \in[1,2]$, one has

$$
\frac{\left(\phi^{\prime}(r)(2 / \alpha)+|\partial r|^{2}\right)^{2}}{3 \phi^{\prime}(r)+\alpha|\partial r|^{2}} \leq\left(\phi^{\prime}(r)+|\partial r|^{2}\right) \leq 4 .
$$

Therefore, if $A_{n} \leq 2 / 5$, then

$$
\begin{aligned}
1- & \frac{2 \phi^{\prime}(r)^{2}\left(\phi^{\prime}(r)(2 / \alpha)+|\partial r|^{2}\right)^{2}}{\left(\phi^{\prime}(r)(-\rho)+\left(\phi^{\prime}(r)^{2}+\alpha \rho\right)|\partial r|^{2}\right)\left(3 \phi^{\prime}(r)+\alpha|\partial r|^{2}\right)} \\
& \geq 1-4 \frac{4 \phi^{\prime}(r)\left(1-A_{n}\right)}{\left(3-4 A_{n}\right)} \\
& \geq 1-\frac{16\left(1-A_{n}\right)}{\left(3-4 A_{n}\right)} \\
& =-\frac{13-12 A_{n}}{\left(3-4 A_{n}\right)} \\
& \geq-\frac{41}{7} .
\end{aligned}
$$


Therefore, if $0 \leq A_{n} \leq 2 / 5$, then

$$
(-\rho) E_{i \bar{j} k \bar{\ell}} \xi_{i} \bar{\xi}_{j} \eta_{k} \bar{\eta}_{\ell} \geq \alpha\left(1-\frac{41}{7} A_{n}^{2}\right)|\xi|^{2}|\eta|^{2} \geq \alpha \frac{11}{175}|\xi|^{2}|\eta|^{2} \geq 0 .
$$

Therefore, the proof of the theorem is complete.

Finally, we will prove part (ii) of Theorem 1.3.

\section{Proof of part (ii) of Theorem 1.3.}

Proof. Let $r(z)=|z|^{2}+\sum_{j=1}^{n} \operatorname{Re} A_{j} z_{j}^{2}-1$ on $D(A)$, and let

$$
\rho(z)=2 r(z)-\frac{1}{2} r(z)^{2}, \quad u(z)=-\log (-\rho(z)), \quad g=\sum_{i, j=1}^{n} \frac{\partial u}{\partial z_{i} \partial \bar{z}_{j}} d z_{i} \otimes d \bar{z}_{j} .
$$

If $A_{n} \leq 2 / 5$ then $|r(z)| \leq 5 / 3$. By (4.10), it is easy to show that $\rho(z)$ is strictly plurisubharmonic in $D(A)$. By Theorem 1.1, we have $\lambda_{1}\left(\Delta_{g}\right)=n^{2}$. By Theorem 4.2, we have $\mathcal{K}_{g} \geq-1$ on $D(A)$. Therefore, the proof of part (ii) of Theorem 1.3 is complete.

\section{Acknowledgment}

S.-Y.L. thanks Peter Li, Zhiqin Lu and Lei Ni for several useful conversations he has had with them during the preparation of this work. He also appreciates the referees who read the manuscript carefully and gives several valuable suggestions for the revision.

\section{References}

[1] S.Y. Cheng, Eigenvalue comparison theorems and its geometric application, Math. Z. 143 (1975), 289-297.

[2] S.-Y. Cheng and S.-T. Yau, On the existence of a complex Kähler metric on noncompact complex manifolds and the regularity of Fefferman's equation, Comm. Pure Appl. Math. 33 (1980), 507-544.

[3] C. Fefferman, Monge-Ampère equations, the Bergman kernel, and geometry of pseudoconvex domains, Ann. Math. 103 (1976), 395-416.

[4] C. Robin Graham, Higher asymptotic of the complex Monge-Ampère equation, Compos. Math. 64 (1987), 133-155. 
[5] L. Ji, P. Li and J. Wang, Ends of locally symmetric spaces with maximal bottom spectrum, J. Reine Angew. Math. 632 (2009), 1-35. 58Jxx (22Exx).

[6] S. Kong, P. Li and D. Zhou, Spectrum of the Laplacian on quaternonic Kähler manifolds, J. Differential Geom. 78 (2008), 295-332.

[7] J. M. Lee and R. Melrose, Boundary behavior of the complex MongeAmpère equation, Acta Math. 148 (1982), 159-192.

[8] P. Li Lecture notes on geometric analysis, Lect. Notes Ser., 6, Research Institute of Mathematics and Global Analysis Research Center, Seoul National University, Korea, 1993.

[9] P. Li, Harmonic functions on complete Riemannian manifolds. Handbook of Geometric Analysis, No. 1, Advanced Lectures in Mathematics, 7, International Press, 2008.

[10] P. Li, and J. Wang, Comparison theorem for Kähler manifolds and positivity of spectrum, J. Differential Geom. 69 (2005), 43-74.

[11] P. Li, and J. Wang, Complete manifolds with positive spectrum II, J. Differential Geom. 62 (2002), 143-162.

[12] P. Li, and J. Wang, Complete manifolds with positive spectrum, J. Differential Geom. 58 (2001), 501-534.

[13] P. Li and J. Wang, Complete manifolds with positive spectrum J. Differential Geom. 58 (2001), 501-534.

[14] S.-Y. Li, Characterization for balls by potential function of KählerEinstein metrics for domains in $\mathbb{C}^{n}$, Comm. Anal. Geom. 13(2) (2005), 461-478.

[15] S.-Y. Li, Characterization for a class of pseudoconvex domains whose boundaries having positive constant pseudo scalar curvature, Comm. Anal. Geom. 17 (2009), 17-35.

[16] S.-Y. Li and M.-A. Tran, Infimum of the spectrum of Laplace-Beltrami operator on a bounded pseudoconvex domain with a Kähler metric of Bergman type, Comm. Anal. Geom. 18 (2010), 375-394.

[17] Q.-K. Lu, On Kähler manifolds with constant curvature, Acta Math. Sinica 16, 344-363 (Chinese); translated as Chinese Math. Acta 8 (1966), 365-384. 
[18] O. Munteanu, A sharp estimate for the bottom of the spectrum of the Laplacian on Kähler manifolds, J. Differential Geom. 84(3) (2010), 611621.

[19] S. Udagawa, Compact Kähler manifolds and the eigenvalues of the Laplacian, Colloq. Math. 56(2) (1988), 341-349.

[20] G. Tian, Canonical metrics in Kähler geometry, note taken by Meike Akveld, Birkläuser Verlag, Basel, Boston, Berlin, 2000.

[21] X. Wang, On conformally compact Einstein manifolds, Math. Res. Lett. 8 (2001), 671-688.

[22] S. M. Webster, Pseudo-Hermitian structure on a real hypersurface, J. Differential Geom. 13 (1978), 25-41.

Department of Mathematics

UNIVERSITY OF CALIFORNIA

IRVINE

CA $92697-3875$

USA

AND

School of Math And Computer Science

FUJiAn NORMAL UNIVERSITY

Fujian, China

E-mail address: sli@math.uci.edu

RECEIVED MAY 5, 2010 